\title{
Duplex ultrasonography arteriography as first-line investigation for peripheral vascular disease
}

\author{
Ting Hway $\underline{\text { Wong }}^{1}$, frCSE, Kiang Hiong $\underline{T a y}^{2}$, FRCR, Mathew G Sebastian ${ }^{1}$, FRCS, FRACS, Seck Guan $\underline{\text { Tan }}^{1}$, FRCS
}

INTRODUCTION The gold standard for evaluation of the lower extremity arterial tree is catheter angiography. Duplex arterial-occlusive imaging or duplex ultrasonography arteriography, a noninvasive technique, is used as the first-line investigation in patients with peripheral vascular disease at our centre. Based on the results of duplex imaging, patients who require angiographic intervention then proceed with simultaneous catheter arteriography and intervention. This study aimed to compare the results of duplex imaging alone as the first-line investigation against the eventual results of catheter angiography, and to assess the impact of the former on patients' clinical outcomes.

METHODS All cases involving patients who underwent duplex imaging followed by angiographic intervention, from May 2008 to February 2009, were discussed at weekly interdisciplinary meetings. Only patients who underwent lower limb imaging were included in the study. Those who were involved in grafts and stent surveillance studies, as well as those with incomplete duplex images were excluded.

RESULTS During the study period, 113 duplex imaging studies of the lower limb followed by percutaneous transluminal angioplasty were performed at our hospital for peripheral vascular disease. The iliac artery was visualised in 40 images, but could not be visualised in 73 images. There was a potential change in management in three cases due to radiological differences between the duplex images and angiography films.

CONCLUSION In our series, duplex imaging was found to be accurate enough to guide initial clinical management of patients with peripheral vascular disease. This modality is the preferred first-line investigation for such patients at our centre.

Keywords: arterial-occlusive imaging, duplex ultrasonography, peripheral vascular disease

\section{INTRODUCTION}

Catheter arteriography is considered the gold standard for the evaluation of the lower extremity arterial tree. However, this technique carries some risks, including allergy, arterial injury, bleeding and contrast-induced nephropathy. ${ }^{(1,2)}$ Duplex arterialocclusive imaging or duplex ultrasonography arteriography, however, is noninvasive and does not carry such risks. The advantages of duplex imaging include characterisation of the vessel wall (such as calcification and suitability for anastomosis), measurement of luminal diameter for therapeutic planning, assessment of chronicity of occlusion, identification of wall pathology (such as aneurysmal vessels with partial thrombosis, or ulcerated/irregular plaques) and haemodynamic information (such as volume and velocity). Some centres that advocate duplex imaging as an alternative to catheter arteriography have used it as the sole preoperative imaging technique for lower limb peripheral vascular disease,,$^{(1,2)}$ although others have advocated alternative forms of three-dimensional imaging. ${ }^{(3,4)}$

In general, patients with peripheral vascular disease managed by the team at the Singapore General Hospital Vascular Studies Unit (SGH VSU) undergo duplex imaging as the firstline investigation. Patients with indications for angiographic intervention (e.g. angioplasty, thrombolysis or stent insertion) subsequently undergo catheter arteriography, followed by intervention at the same session. The SGH VSU undertakes all arterial imaging of the distal extremities for peripheral vascular disease. From May 2008, all duplex imaging requests at the hospital have been screened by vascular surgeons to ensure that only patients who require interventions would undergo imaging. Percutaneous angioplasty is the preferred interventional procedure for peripheral vascular disease at SGH.

The goal of this study was to audit the predictive value of duplex ultrasonography arteriography findings, using catheter arteriography as the gold standard, and assess the impact of any differences between the findings of these modalities on patients' clinical outcomes.

\section{METHODS}

All cases involving patients who underwent duplex imaging and subsequent angiographic intervention from May 2008 to February 2009 were discussed and the images rated at weekly multidisciplinary VSU meetings. During the study period, 113 angiographic interventions with duplex imaging performed in the SGH VSU were rated at these meetings. For the purposes of this study, only patients who had undergone lower limb imaging for peripheral vascular disease were included. Patients under graft and stent surveillance studies, as well as those with incomplete duplex images (unable to visualise all five segments of the lower limb arterial tree), were excluded from the study.

The weekly VSU meetings were attended by interventional radiologists, vascular surgeons and VSU vascular technologists. Duplex images were compared with angiography films that

${ }^{1}$ Department of General Surgery, ${ }^{2}$ Department of Radiology, Singapore General Hospital, Singapore

Correspondence: Dr Ting Hway Wong, Consultant, Department of General Surgery, Singapore General Hospital, Outram Road, Singapore 169608. wong.th@doctors.org.uk 


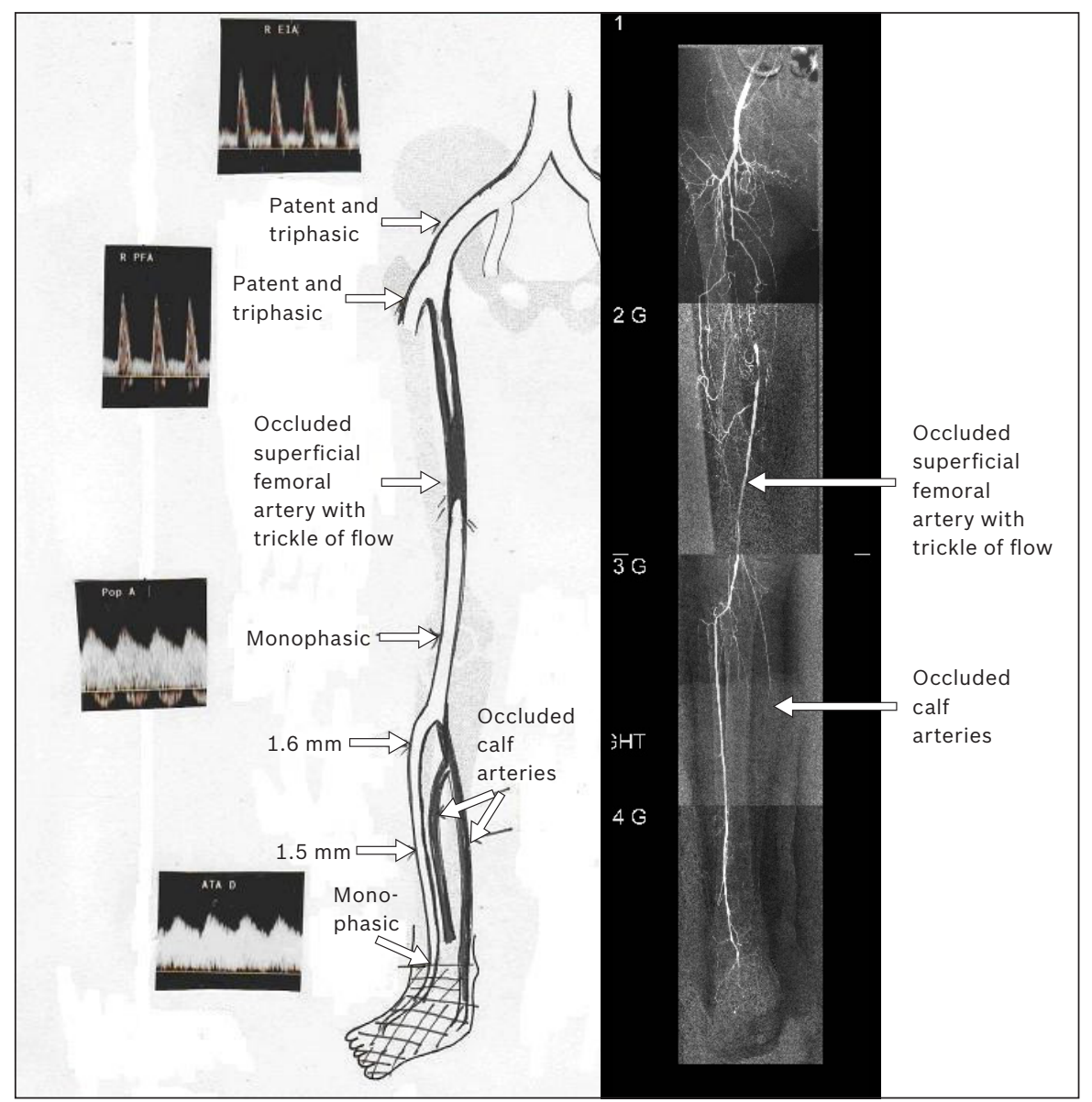

Fig. 1 Angiography images vs. duplex images of the right leg in the same patient.

were captured during angioplasty and graded. The grading system used accords one point to each segment of the lower limb arteries when a positive correlation between the two modalities is shown, i.e. findings of the duplex image matches the corresponding angiographic segments. All the scorers had at least five years of experience in their respective fields. A consensus grade was given during the meetings. The lower limb arterial segments selected for comparison in the anatomical grading system adopted by this study included the: (a) iliac; (b) femoropopliteal; (c) posterior tibial; (d) peroneal; and (e) anterior tibial and dorsalis pedis arteries. A simple five-point grading system was used to compare the duplex images with the angiography films, graded according to segment and degree of stenosis. For example, if a lesion was seen in one segment as $50 \%-75 \%$ occluded on duplex imaging but $>75 \%$ occluded on digital subtraction angiography (DSA), while all other segments were in agreement, the grading would be $4 / 5$. Similarly, lesions that were $>75 \%$ occluded on duplex imaging but totally occluded on DSA were also considered to be discrepant. If multiple lesions were seen on DSA and significant lesions were missed on duplex imaging, they were also considered discordant. Segments that were considered normal on one and graded mildly occluded $(<50 \%)$ on the other test were considered concordant. A five-point grading system was deemed to be simple to use and adequate for the purposes of the present study. We resisted increasing the number of arterial segments to be correlated, as that would have made the study more complex and unwieldy, though at the expense of slight limitations in accuracy. In addition to grading, we also noted down whether the radiological differences between the findings of duplex imaging and DSA would have changed the patient's management.

This was a retrospective study that compared catheter arteriography films taken during therapeutic angiography with pre-angiography duplex ultrasonography reports. The duplex imaging report was deemed to be accurate if the findings correlated with those of angiographic studies and did not warrant a change in the patient's clinical management. As seen in the published literature of other large vascular units, 'accuracy' suggests a positive correlation in excess of $90 \%$ between the two modalities and less than $1 \%$ of cases revealing differences with implications for changes in the patient's clinical management. (5,6) $^{(5,}$

\section{RESULTS}

During the study period, a total of 113 lower limb duplex imaging studies performed for peripheral vascular disease were followed up with percutaneous transluminal angioplasty. Out of these, only 40 angiograms had fully visualised iliac arteries on catheter angiography; thus only 40 of these images could be fully graded. In the remaining images, which had been approached via downhill puncture, the iliac arteries were not fully visualised. 38 images were graded 5/5 on the five-point grading system 
(Fig. 1), 49 were graded 4/4, 2 were graded $4 / 5$ and 24 were graded 3/4. Of the 26 images graded 4/5 and 3/4, 23 did not have radiological differences between the duplex images and angiography films that would have led to any change in patient management. The above findings are summarised in Table I.

A change in patient management following angiography was required for only three images. In one of these images, the posterior tibial artery was seen to have only a proximal diseased segment on duplex imaging, whereas angiography showed that it was completely obliterated and therefore would not have been a suitable target for angioplasty. For the remaining two images, the iliac arteries were seen as triphasic on Doppler images, but had significant stenosis requiring angioplasty on catheter angiography. The sites of disagreement were almost equally distributed between the femoropopliteal $(n=9)$ and infragenicular $(n=15)$ segments. Of the 40 iliac segments visualised, 38 were in agreement, indicating a concordance of over $90 \%$ for individual segments in our study (Table II).

113 imaging studies in our series were performed on 110 patients; three patients had bilateral lower limb duplex imaging followed by bilateral angiographic interventions. The 110 patients had the following comorbidities: diabetes mellitus $(n=103$, $93.6 \%)$, ischaemic heart disease $(n=75,68.2 \%)$, end-stage renal failure ( $\mathrm{n}=35,31.8 \%)$, renal impairment without dialysis requirement $(n=21,19.1 \%)$, chronic renal disease $(n=56,50.9 \%)$ and hypertension ( $\mathrm{n}=102,92.7 \%$ ). Indication for angiographic interventions was Fontaine grade IV in all except two patients. One patient had lifestyle-limiting claudication not relieved by exercise therapy (Fontaine grade II), while another patient had claudication that progressed to rest pain (Fontaine grade III). Both of these patients had grade 5/5 duplex images. Angiographic interventions were performed after a median of 8 (range 1-65) days following duplex imaging. The difference in the median time interval for images rated 5/5 (mean 8 days; range $1-65$ days) and those rated 4/5 (mean 7.5 days; range 2-36 days) was not statistically significant. For five patients who underwent angioplasty, the time interval between duplex imaging and angiography/angioplasty was more than 30 days. Three patients had dry gangrene at the time of initial duplex imaging, and all images in these three patients were rated 5/5. Delays were caused by noncompliance with cardiac treatment causing life-threatening cardiac complications $(n=1)$ and default from follow-up $(n=4)$.

\section{DISCUSSION}

In our series, duplex imaging was generally accurate enough to guide initial clinical management, and was the preferred first line of investigation for peripheral vascular disease. Regular audit and feedback to clinicians, technologists and radiologists during our weekly meetings was crucial in maintaining such a high level of accuracy. Other centres have reported similarly good results for concordance between duplex imaging and catheter angiography in patients with peripheral
Table I. Anatomical grading system comparing duplex images to catheter angiography images.

\begin{tabular}{lc}
\hline Image $(\mathbf{n}=\mathbf{1 1 3})$ & No. of patients $^{*}$ \\
\hline With iliac artery visualised $^{*}$ & 40 \\
Score $5 / 5$ & 38 \\
Score $4 / 5$ & 2 \\
Without iliac artery visualised $^{+}$ & 73 \\
Score 4/4 & 49 \\
Score 3/4 & 24 \\
\hline
\end{tabular}

${ }^{\star}$ Graded out of $5 .{ }^{\dagger}$ Graded out of 4 .

Table II. Sites of disagreement observed on comparing duplex imaging to catheter angiography.

\begin{tabular}{|c|c|c|}
\hline Site of disagreement & $\begin{array}{c}\text { No. of } \\
\text { patients }\end{array}$ & $\begin{array}{c}\text { Concordance } \\
(\%)\end{array}$ \\
\hline Iliac segment* & 2 & 95.0 \\
\hline $\begin{array}{l}\text { Femoropopliteal segment }{ }^{+} \\
\text {Femoral } \\
\text { Popliteal }\end{array}$ & $\begin{array}{l}9 \\
6 \\
3\end{array}$ & $\begin{array}{l}92.0 \\
94.7 \\
97.3\end{array}$ \\
\hline $\begin{array}{l}\text { Infragenicular segment } \\
\text { Anterior tibial artery } \\
\text { Posterior tibial artery } \\
\text { Peroneal artery }\end{array}$ & $\begin{array}{r}15 \\
6 \\
6 \\
3\end{array}$ & $\begin{array}{l}86.7 \\
94.7 \\
94.7 \\
97.3\end{array}$ \\
\hline
\end{tabular}

* Out of 40 images. ${ }^{\dagger}$ Out of 113 images

vascular disease. ${ }^{(5,6)}$ However, a few factors have complicated the direct comparison of our results with those from other centres.

First, various studies have used different definitions of 'test agreement'. For instance, Hingorani et $\mathrm{al}^{\left({ }^{(5)}\right.}$ distinguished only between normal and abnormal test results, and made no distinction between the degree of stenosis. Their study showed 95\% accuracy for tibial vessels and 96\% accuracy for aortoiliac and femoropopliteal segments. ${ }^{(5)}$ In our series, for vessels distal to the femoral pulse, there was $100 \%$ test agreement between duplex imaging and catheter arteriography in terms of abnormal vs. normal vessel findings. The difference seen in our test results was only with regard to the degree of abnormality. Similarly, in a study by Favaretto et al, ${ }^{(6)}$ the agreement coefficient (kappa) was defined as stenosis that was categorised as a binomial variable $(0,1)$ based on the presence of stenosis of $>70 \%$ luminal diameter reduction. Such a definition has good relevance for therapeutic practice, since angiographic intervention is usually indicated when stenosis is $\geq 70 \%$. Based on this definition, Favaretto et al reported $62 \%-70 \%$ accuracy for the femoral, popliteal and iliac segments, but only $25 \%$ accuracy for the tibial arteries. ${ }^{(6)}$ By the same definition, the results of our series compared favourably with Favaretto et al's study by translating into $86.7 \%$ overall accuracy for the tibial vessels, 92\% accuracy for the femoropopliteal segments and $95 \%$ accuracy for the iliac vessels.

In our series, there were two instances where duplex imaging showed triphasic iliac arteries, while DSA showed significant stenosis. In our clinical practice, if a patient does not have a palpable femoral pulse on clinical examination, the usual procedure is to perform a computed tomography angiography (CTA) of the iliac vessels to complement the findings of lower 
Table III. Comparison of the cost of various imaging modalities being used as first-line investigation for peripheral vascular disease.

\begin{tabular}{ll}
\hline Imaging modality & Cost (SGD) $^{*}$ \\
\hline Duplex ultrasonography arteriography & 200 \\
Computed tomography angiography & 900 \\
Magnetic resonance angiography & 1,600 \\
Catheter angiography & $1,500+1,000^{+}$ \\
\hline
\end{tabular}

* Prices are approximate. Taken at time of study in $2009 .{ }^{\dagger}$ The cost is for intervention (angioplasty).

SGD: Singapore dollars

limb duplex imaging prior to angiographic intervention. This was the approach adopted for the two images in our series that showed discordant results for the iliac vessels.

Second, the inclusion criteria for patients differed among various studies. $98 \%$ of patients in our series were classified as Fontaine grade IV (tissue loss or gangrene) at the time of the imaging studies; only one patient underwent angiography for rest pain, while another underwent angiography due to claudication. In contrast, Hingorani et al reported that $19 \%$ and $9 \%$ of their 61 patients underwent angiography for rest pain and claudication, respectively. ${ }^{(5)} \mathrm{A}$ larger study by these same authors found that $40 \%$ of patients were classified as Fontaine grade IV at the time of the imaging studies, with only $20 \%$ of patients undergoing angiography for rest pain, $7 \%$ for acute ischaemia and the remaining for claudication or popliteal aneurysm. ${ }^{(7)}$ The latter study by Hingorani et al reported that $10 \%$ of patients required angiography in addition to duplex ultrasonography; ${ }^{(7)}$ this rate was higher than that found in our study. Meanwhile, in Favaretto et al's series of 49 patients, patients either had tissue loss at the time of imaging (25\%), claudication $(50 \%)$ or critical ischaemia $(25 \%){ }^{(6)}$ In a study by Ouwendijk et al, less than $20 \%$ of patients underwent imaging for critical ischaemia. ${ }^{(4)}$

These results reflect the divergence of treatment practices adopted by various studies, as well as differences in disease demography and patient expectations. At our institution, the first-line therapy of choice for claudication is intensive walk exercise therapy. The comorbidity profile of patients in our series was also somewhat different from those seen in other centres. While the incidence of diabetes mellitus in our series is similar to that in Hingorani's study ${ }^{(5)}(94 \%$ vs. $86 \%$ ), our series has a much higher incidence of hypertension ( $92.7 \%$ vs. $59 \%)$ and renal failure (32\% vs. $15 \%$ ). In Ouwendijk et al's trial, only 50\% of patients had hypertension, $<30 \%$ had diabetes mellitus and $<10 \%$ of patients had renal disease. ${ }^{(4)}$ While such differences in the comorbidity profiles of patients may not have affected the accuracy of duplex imaging, it may be indicative of the more advanced stages of vascular disease in our patient population.

In conclusion, the authors concur with researchers who have proposed that duplex imaging is a safe and accurate first-line investigation for peripheral vascular disease. It is also more economical compared to other imaging modalities (Table III). While some authors have advocated $\mathrm{CTA}^{(8)}$ or magnetic resonance angiography for lower limb peripheral arterial disease, ${ }^{(3)}$ CTA is used at our institute only in selected cases and solely to complement duplex imaging, similar to the practice in other centres. ${ }^{(9)}$ Currently, duplex-guided angioplasty is also performed at our centre for patients at high risk of contrast nephropathy, and we hope to report related results in the future. We conclude that our experience with duplex imaging as a first-line investigation for peripheral vascular disease suggests that it is adequate for arriving at appropriate decisions regarding the management and intervention of patients with peripheral vascular disease.

\section{ACKNOWLEDGEMENTS}

We would like to thank the Singapore General Hospital Vascular Studies Unit for their assistance with this study.

\section{REFERENCES}

1. Ascher E, Hingorani A, Markevich N, Schutzer R, Kallakuri S. Acute lower limb ischemia: the value of duplex ultrasound arterial mapping (DUAM) as the sole preoperative imaging technique. Ann Vasc Surg 2003; 17:284-9.

2. Ascher E, Mazzariol F, Hingorani A, Salles-Cunha S, Gade P. The use of duplex ultrasound arterial mapping as an alternative to conventional arteriography for primary and secondary infrapopliteal bypasses. Am J Surg 1999; 178:162-5.

3. Collins R, Burch J, Cranny G, et al. Duplex ultrasonography, magnetic resonance angiography and computed tomography angiography for diagnosis and assessment of symptomatic, lower limb peripheral arterial disease: systematic review. BMJ 2007; 334:1257-61.

4. Ouwendijk R, de Vries M, Stijnen T, et al. Multicenter randomized controlled trial of the costs and effects of noninvasive diagnostic imaging in patients with peripheral arterial disease: the DIPAD trial. AJR Am J Roentgenol 2008; 190:1349-57.

5. Hingorani A, Ascher E, Marks N. Preprocedural imaging: new options to reduce need for contrast angiography. Semin Vasc Surg 2007; 20:15-28.

6. Favaretto E, Pili C, Amato A, et al. Analysis of agreement between Duplex ultrasound scanning and arteriography in patients with lower limb artery disease. J Cardiovasc Med (Hagerstown) 2007; 8:337-41.

7. Hingorani AP, Ascher E, Marks N, et al. Limitations of and lessons learned from clinical experience of 1,020 duplex arteriography. Vascular 2008; 16:147-53.

8. Met R, Bipat S, Legemate DA, Reekers J A, Koelemay, MJ. Diagnostic Performance of Computed Tomography Angiography in Peripheral Arterial Disease: A Systematic Review and Meta-analysis. JAMA 2009; 301:415-24.

9. Bradbury AW, Adam DJ. Diagnosis of peripheral arterial disease of the lower limb. BMJ 2007; 334:1229-30. 